\title{
Double Appendixes: A Rare Case Report
}

\author{
Markos Markou ${ }^{1}$, Rodopi Sotiropoulou ${ }^{1}$, Sebach Perente ${ }^{1}$, Ioanna-Triada Kougioumtzi ${ }^{1}$, \\ Soultana Foutzitzi ${ }^{2}$, paschalis chatzipantelis ${ }^{1}$, and sotirios botaitis ${ }^{3}$ \\ ${ }^{1}$ Democritus University of Thrace School of Health Sciences \\ ${ }^{2}$ Radiologist \\ ${ }^{3}$ Democritus University of Thrace Department of Medicine
}

November 25, 2021

\begin{abstract}
Anomalies of the appendix are rare, and one of the rarest is the double appendixes. Most anomalies of the appendix are observed in adults and are discovered incidentally during surgery that does not primarily involve the appendix. It is usually missed, often with life-threatening consequences.
\end{abstract}

\section{Double Appendixes: A Rare Case Report}

Markou Markos ${ }^{1}$, Sotiropoulou Rodopi ${ }^{1}$, Perente Sebach $^{1}$, Kougioumtzi Ioanna ${ }^{1}$, Foutzitzi Soultana ${ }^{2}$, Chatzipantelis Paschalis ${ }^{3}$, Botaitis Sotirios ${ }^{1}$

${ }^{1} 1$ st Department of Surgery, Alexandroupoli University Hospital, School of Medicine, Democritus University of Thrace, GR-68100 Alexandroupolis, Greece

${ }^{2}$ Radiology Department, Alexandroupoli University Hospital, School of Medicine, Democritus University of Thrace, GR-68100 Alexandroupolis, Greece

${ }^{3}$ Department of Pathology, Alexandroupoli University Hospital, School of Medicine, Democritus University of Thrace, GR-68100 Alexandroupolis, Greece

Corresponding author:

Botaitis Sotirios

Associate Professor of Surgery

Alexandroupoli University Hospital,

School of Medicine, Democritus University of Thrace,

68100 Alexandroupolis, Greece

$\mathrm{T}+30-2551343224$

E smpotait@med.duth.gr

Key words: Double appendixes, appendiceal duplication, anatomical variations of vermiform appendix, appendicitis

Declarations of interest: none 
Written informed consent was obtained from the patient to publish this report in accordance with the journal's patient consent policy

\section{Double Appendixes: A Rare Case Report}

\section{Key Clinical Message}

Every surgeon must be aware of the anatomical variations of the vermiform appendix. Duplication of the appendix should be considered in the differential diagnosis for patients with pain in the right iliac fossa.

\section{Introduction}

Anomalies of the appendix are rare, and one of the rarest is the double appendixes. Most anomalies of the appendix are observed in adults and are discovered incidentally during surgery that does not primarily involve the appendix. There are many types of double appendixes, with some of the cases including duplication of other organs. It is usually missed unless a person undergoes a surgery that reveals it, often with lifethreatening consequences. Herein, we present a rare case of a patient with duplication of the vermiform appendix having acute appendicitis of both appendixes.

\section{Case Report}

A 30-year-old female patient with a height of $1.62 \mathrm{~m}$ and weight of $110 \mathrm{~kg}$ (BMI 41.91) having pain in the lower abdomen was admitted to the emergency department. The previous day, symptoms started with pain in the epigastrium. As time went on, the pain was located around the navel and finally settled in the right iliac fossa. The patient did not report having nausea, episodes of vomiting, or fever at home. The patient's bowel habits were unaltered, and she reported loss of appetite the previous two days.

The physical examination raised the suspicion of appendicitis since a positive McBurney's sign was found - right lower abdominal quadrant pain - and rebound tenderness, indicating peritoneum irritation. The blood test results revealed leucocytosis, with WBC 12.730/ $\mathrm{mm}^{3}$ (66.4\% Neut, $23.6 \%$ Lym, $8.1 \%$ Mono) and increased levels of the C-reactive protein (2.06). Our patient had an Alvarado score 9 [1]. Additionally, neither the urine analysis nor the abdominal X-ray revealed any findings.

Afterward, an examination of the abdomen was performed with an ultrasound. The coarse examination revealed no particular findings. The graded compression technique was then performed using the $8 \mathrm{MHz}$ probe set above the position of maximum sensitivity with gradually increasing pressure exerted to displace the normal supernatant gas. The appendix was identified (Figure 1) with the blind end of the appendix arising from the base of the caecum. The appendix was dilated (diameter $1.5 \mathrm{~cm}$ ) in target appearance (axial section) [a], was non-compressible when compression was applied [b] and had hyperechoic appendicolith with posterior acoustic shadowing and periappendiceal fluid collection (white arrow) [c]. After the application of the coloured Doppler, it showed intense vascularization of the wall as an image of mural hyperaemia [d].

The patient was hemodynamically stable but, during admittance, presented with a low-grade fever $\left(37.5{ }^{\circ} \mathrm{C}\right)$. After reviewing the test results, a decision was made to perform an open appendectomy via a McBurney's incision.

Intraoperative findings included a mild quantity of free fluid in the right iliac fossa and an inflamed appendix. Surprisingly, another thin, mildly inflamed appendix was found when the appendectomy was completed (Figure 2). 
Both the appendices could be separated at the bases and were ligated individually. Our case presented a B2 type appendiceal duplication [2]. An inspection of the small bowel for the presence of Meckel's diverticulum followed, which was negative. Histopathological examination of the surgical specimen confirmed the clinical findings. Both specimens showed identical features: appendiceal mucosa with extensive transmural chronic, active inflammation associated with suppurative peritonitis (Figure 3a,b). The patient had an uneventful recovery and was discharged on the sixth postoperative day.

\section{Discussion}

The first person to describe a case of appendiceal duplication was Picoli in 1892. Its prevalence worldwide is $0.004 \%$ to $0.009 \%$ [3]. In a worldwide search of scientific literature, most references to appendiceal duplication are found in case reports, indicating the rarity of this condition. In 1936, Cave proposed a classification system based on their anatomical location [4], and in 1963, Wallbridge revised this classification, and the modified Cave-Wallbridge classification was created [2]. Minor modifications were made until 1993 when Biermann suggested the following classification, which is used today:

- Type A: Single caecum with one appendix exhibiting partial duplication.

- Type B: Single caecum with two obviously separate appendixes.

- B1: The two appendixes arise on either side of the ileocaecal valve in a 'bird-like' manner.

- B2: In addition to a normal appendix arising from the caecum at the usual side, there is also a second, usually rudimentary, appendix arising from the caecum along the lines of the taenia at a varying distance from the first.

- B3: The second appendix is located along the taenia of the hepatic flexure of the colon.

- B4: The location of the second appendix is along the taenia of the splenic flexure of the colon.

- Type C: Double caecum, each bearing its own appendix and associated with multiple duplication anomalies of the intestinal tract as well as the urinary tract.

- Type D: Horseshoe anomaly of the appendix [5].

Our case presented a B2 type appendiceal duplication, which is the most common type. According to a study by Nageswaran et al. [6], there are no associated congenital abnormalities in this type of duplication; concealed duplication is confirmed only intraoperatively.

Type B2 duplication is the most common variation of anatomy and the most difficult to identify. Difficulty in identification is because the appendix that arises from the convergence of the taenia is retrocaecal and out of sight. Moreover, if an inflamed, anteriorly placed appendix is found, the retrocaecal space is not usually explored. It is considered that approximately $37 \%$ of patients with duplication present with inflammation of both appendices at the time of operation; therefore, they may not recover postoperatively as expected. If signs of inflammation are present along the right paracolic gutter when the surgeon identifies an anteriorly placed appendix, careful examination of the caecal pole and retrocaecal space should be subsequently performed.

Some extremely rare cases are described, such as 'the triple appendix' [7], which cannot include the existing types. In 1986, Alvarado [1] suggested a clinical diagnostic tool which considers the patient's signs and symptoms as well as some laboratory values. It is used for stratifying the risk of appendicitis being present (Table 1). A score of 5 or 6 is compatible with the diagnosis of acute appendicitis; a score of 7 or 8 indicates probable appendicitis; and a score of 9 or 10 indicates a very probable appendicitis. The Alvarado score is considered to have high sensitivity and low specificity; therefore, it is useful in "catching" appendicitis. However, the score is less effective for stratifying the risk of appendicitis in children [8]. After some years, this score was modified for patients 3-18 years old, and the Paediatric Appendicitis Score was created and implemented.

\section{Table 1}

Alvarado Score 


\begin{tabular}{ll}
\hline Feature & Score \\
\hline Symptoms & 1 \\
Migration of pain & 1 \\
Anorexia & 1 \\
Nausea & 2 \\
Signs & 1 \\
Tenderness on the right lower quadrant & 1 \\
Rebound pain & 2 \\
Elevated temperature & 1 \\
Laboratory Values & 10 \\
Leucocytosis & \\
Shift of white blood cell count to the left & Total
\end{tabular}

To briefly summarize the embryology of the normal appendix, during the fifth foetal week, a bud at the junction of the small and large bowel develops and undergoes rapid growth into a pouch. The proximal end of this pouch starts growing differentially to give rise to the caecum. The appendix first appears at the eighth week of gestation as an outpouching of the caecum. Then, it gradually rotates to a more medial location following the respective rotation of the gut, which results in the fixation of the caecum in the right lower quadrant [9]. The normal embryogenesis of the appendix is well-known. However, there are no data regarding the causes of its duplication, and malrotation does not seem to be responsible for the pathophysiology of the condition. Cave [4] tried to explain the pathogenesis of duplication. To achieve this, he concluded with two theories: a) the persistence of a transient embryological structure and b) incidental appendiceal duplicity to a more general affection of the primitive midgut. However, even though Cave's theories may explain some types of duplication, they are inadequate to explain all types reported.

It is difficult to diagnose double appendixes using routine imaging examinations. Abdominal ultrasound and computed tomography (CT) are the main methods available. However, these image examinations - mainly the CT scan - are usually not included in the routine workup of otherwise healthy patients with pain in the right iliac fossa. In cases where the patient is a child, the situation is even more challenging since a CT is avoided as the initial imaging choice due to the risk of radiation exposure. The CT has been reported to identify duplication of the appendix, especially in cases where both appendixes are significantly inflamed $[10]$.

\section{Conclusion}

The duplication of the appendix is rare, and its treatment is challenging. Therefore, every surgeon must be aware of the anatomical variations of the vermiform appendix, and the caecum requires routine visual inspection to ensure that there are no appendiceal anomalies. Duplication of the appendix should be considered in the differential diagnosis for patients with pain in the right iliac fossa, even if the patient reports previously having an appendectomy, especially if a CT scan has not been performed. Misdiagnosis of this situation can lead to life-threatening complications for the patient and medicolegal consequences.

\section{Authors contributions}

This work was carried out in collaboration between all authors. Authors M. Markou and R. Sotiropoulou examined the patient, established diagnosis and participated in the surgery. Author M. Markou wrote the first draft. Author R. Sotiropoulou helped in writing. Author S. Perente participated in the surgery and 
reviewed the manuscript. Author I. Kougioumtzi reviewed the manuscript. Authors S. Foutzitzi and P. Chatzipantelis established diagnosis and searched the literature. Author S. Botaitis participated in the surgery and wrote the final version of the manuscript. All authors read and approved the final manuscript.

\section{Acknowledgments}

None.

\section{References}

1. Alvarado A. A practical score for the early diagnosis of acute appendicitis. Ann Emerg Med 1986;15:557-64.

2. Wallbridge PH. Double appendix. British Journal of Surgery 1963;50:346-7.

3. Collins D. A study of 50,000 specimens of the human vermiform appendix. Surgical Gynecological Obstetrics 1955;101:437-46.

4. Cave AJE. Appendix vermiformis duplex. J Anat 1936;70:283-92.

5. Mesko TW, Luso R, Breitholtz T. Horseshoe anomaly of the appendix: a previously undescribed entity. Surgery 1989;106:563-566.

6. Nageswaran H, Khan U, Hill F, Maw A. Appendiceal Duplication: A Comprehensive Review of Published Cases and Clinical Recommendations. World Journal of Surgery 2017;42(2):574-581.

7. Uriev L, Maslovsky I, Mnouskin Y, Ben-Dor D. Triple-barreled type of appendiceal triplication. Annals of Diagnostic Pathology 2006;10(3):160-1.

8. Ohle R, O'Reilly F, O'Brien KK, Fahey T, Dimitrov BD. The Alvarado score for predicting acute appendicitis: a systematic review. BMC Med 2011;9:139.

9. Schumpelick V, Dreuw B, Ophoff K, Prescher A. Appendix and cecum. Embryology, anatomy, and surgical applications. Surgical Clinics of North America 2000;80(1):295-318.

10. Chew DK, Borromeo JR, Gabriel YA, Holgersen LO. Duplication of the vermiform appendix. Journal of Pediatric Surgery 2000;35(4):617-8.

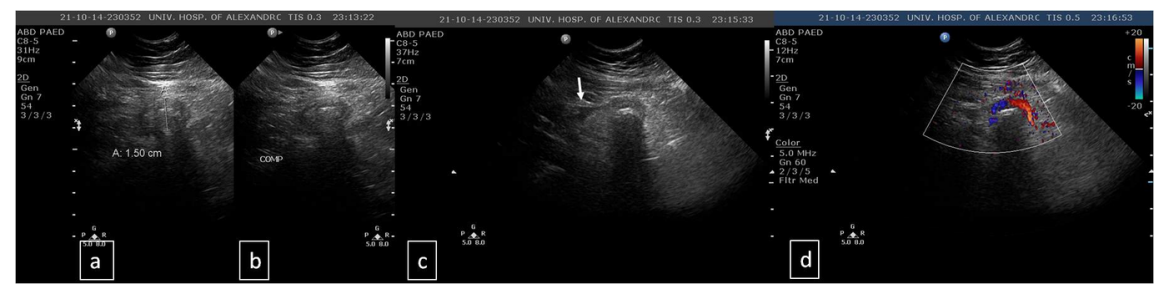




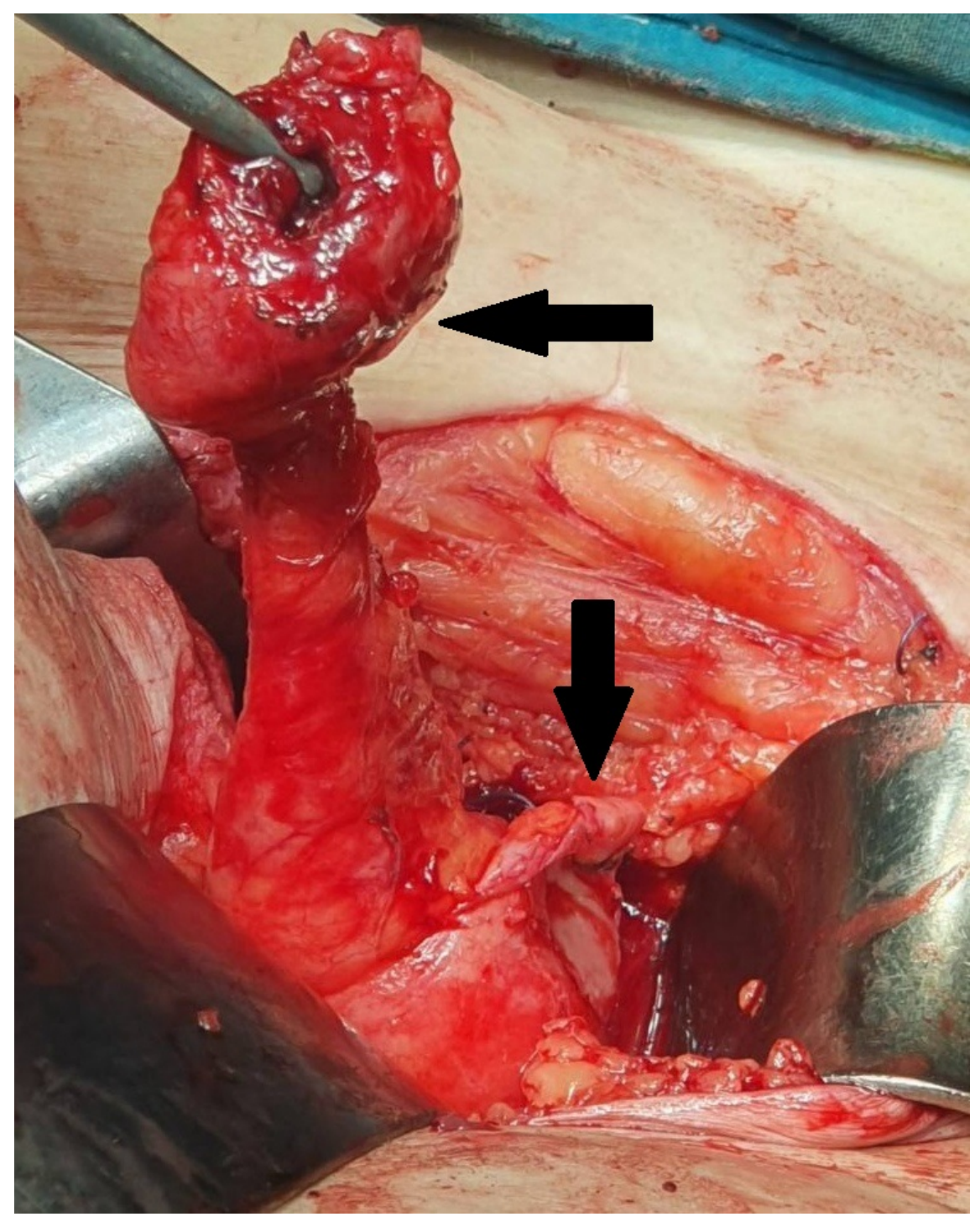



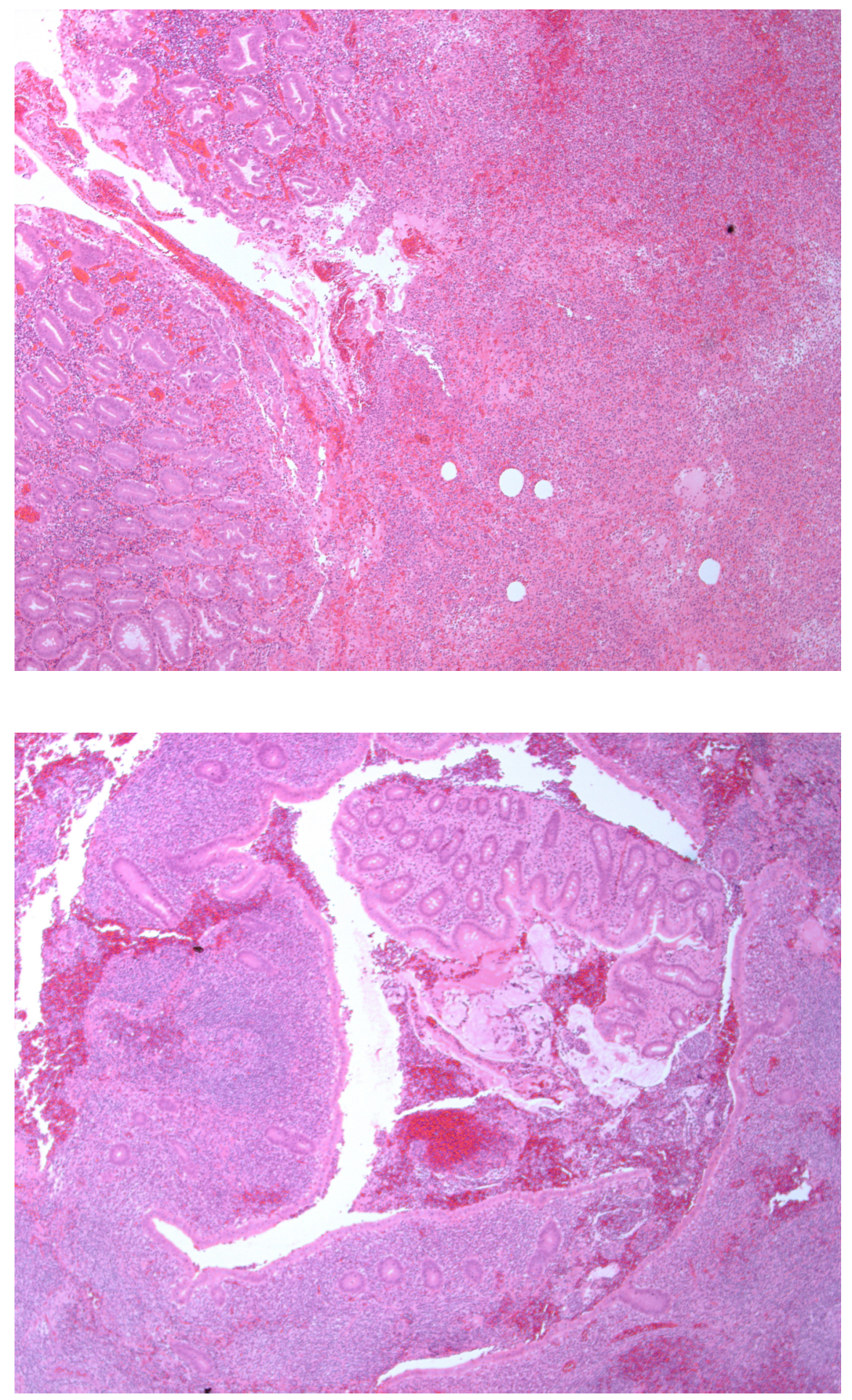